Iraqi Journal of Information and Communications Technology(IJICT)

Conference Series: The $1^{\text {st }}$ Conference of Applied Researches in Information Engineering(ARIE2021), 2021

ISSN:2222-758X e-ISSN:2789-7362

\title{
HIGH-QUALITY LOW BITRATE VOICE CODEC FOR TRANSMISSION OVER ADVANCED LTE
}

\author{
Noor N. Edan ${ }^{1}$, Nasser N. Khamiss ${ }^{2}$ \\ 1,2 College of Information Engineering, Al-Nahrain University, Baghdad, Iraq \\ \{noor.naser, nasser\} @ coie-nahrain.edu.iq ${ }^{1,2}$ \\ Received:4/5/2021, Accepted:28/5/2021
}

\begin{abstract}
In mobile communication systems bit-rate reductions while maintaining an acceptable voice quality is necessary to achieve efficiency in channel bandwidth utilization and users satisfaction. As Long-Term Evolution (LTE) converging towards all-IP solutions and supporting VOIP service, the voice signals are converted into coded digital bit-stream and sent over the network. This paper proposes the implementation of a codebook excited linear prediction (CELP) voice codec algorithm based on two source rates of low 9.6Kbps and medium 16Kbps for achieving a perceptible level of voice quality, while efficiently using available bandwidth during the transmission over advanced LTE. The architecture of the proposed CELP codec model is implemented to decompose the voice signal into a set of parameters that characterize each particular frame at the encoder part, these parameters are quantized and encoded for transmission to the decoder. The investigation showed that the configuration of the link and the applied CELP codec mode mainly influence the obtained voice capacity and quality. For voice quality evaluation, the examination showed better voice quality obtained with a low value of noise weighting factor and for a higher codec rate. The quantifying also shows that the voice quality can be traded for the enhanced capacity since the low rate codec of $9.6 \mathrm{kbps}$ provide better voice capacity at the cost of inferior voice quality compared to the high rate codec of 16kbps. Also, this paper is achieved increasing in the capacity gain to a saturated value during the configuration of the system with a higher channel quality indicator (CQI) index. For transmit diversity (TD), about 500 and up to 1000 users per cell over $5 \mathrm{MHz}$ and $10 \mathrm{MHz}$ bandwidth respectively are obtained. For Open-Loop Spatial Multiplexing (OLSM) up to 1000 and 2000 users per cell over are obtained over 5MHz and $10 \mathrm{MHz}$ channel bandwidth respectively.
\end{abstract}

keywords: CELP, LTE, CQI, VOIP, TD, OLSM.

\section{INTRODUCTION}

In Global mobile networks, voice service has been always considered as the essential revenue generator for mobile operators as it is the main required service by subscribers. The increasing gap between capacity and demand poses an urgent call for novel network technologies that allow mobile operators to improve performance on a cost-effective basis [1] . Long-Term Evolution (LTE) is an attractive broadband wireless standard that is designed to deliver enhanced multimedia, voice and video services to end-users [2] . LTE derived as a complete IP cellular network that offered wideband radioaccess technology called Evolved-Universal Terrestrial Radio Access Network (E-UTRAN) with evolved Node B (eNBs) [3]. Advanced LTE developed as an extension to LTE network with an enhanced radio-access technology [4] , [5] . During voice communication over Advanced LTE, compression with an efficient voice codec is an essential requirement to managing the available bandwidth by transforming the voice signal at the application layer into a compressed digital stream of data bits [6] . During the transmission of encoded voice bits over the downlink physical layer, eNB assign LTE Resource Blocks (RBs) for carrying the control information and User Equipment's (UE'S) voice bits over Transmission Time Intervals (TTIs) [7]. As E-UTRAN in LTE is purely packet-switched (PS) radio access technology, therefore the voice service is delivered through Voice over Internet Protocol (VOIP) based mechanisms to enable the operators to roll out packetized voice for LTE networks with greater voice capacity and higher voice quality [5]. As LTE-A supporting VOIP service, the number of assigned Resource Blocks (RBs) for each subscriber will be affected by many aspects. Subscriber's encoded voice data 
Iraqi Journal of Information and Communications Technology(IJICT)

Conference Series: The $1^{\text {st }}$ Conference of Applied Researches

in Information Engineering(ARIE2021), 2021

ISSN:2222-758X

e-ISSN:2789-7362

size is considered as the main issue. Therefore, a compression capability to save network bandwidth and increase system capacity became essential. Moreover, a new challenge arises from the necessity of providing a perceptible level of voice quality to end-users. One of the most effective and broadly used codec algorithms for mobile communication is the CELP algorithm and it is considered as a generic algorithm that affords highly efficient and high-quality speech coding [8] . With the CELP algorithm, exploiting the redundancy in voice traffic can attain a better compression at bit rates between 4.8 and 16 kbps [9] , [10]. The most important driving forces that enable CELP for increasing system capacity and preserve a certain quality is the hybrid process, analysis by synthesis and voice signal parameterization based codec [9] , [11]. Another attractive approach in CELP is the use of a codebook that incorporates all the excitation sequences that are to be searched for the selection of the optimum sequence for encoding short voice segments in the encoder and only the selected sequence index is to be transmitted [9]. This method provides data bit compression, in addition to accomplishing highquality voice and bandwidth optimization [12] . During the paper's work time, many types of research in recent years are studied. Mohammed et al, in 2015 [13] presented VoIP options on LTE networks and concluded that the option of VoIP on LTE can offer minimum service guarantees that require more than the best effort as the traditional IP networks. Jage and Upadhya , in 2016 [10] implemented the CELP and Mixed Excitation Linear Prediction(MELP) coding techniques of speech. The analysis of the CELP speech coding technique showed that this technique is an improvement to a coder called Linear Predictive Coder (LPC) and CELP is an efficient coding technique having a bit rate in the range of 9.6 kbps to $16 \mathrm{kbps}$. The analysis was performed based on subjective and objective tests like MSE, Mean Opinion Score (MOS) and SNR. The study was showed that, at the values of perceptually weighted constant of 0.5 , the reconstruction of the speech signal is better and audio quality and intelligibility are also maintained. Joshi et al, in 2018 [12] examined the performance analysis of CELP coding at 16 and $9.6 \mathrm{kbps}$ bit rates with MSE and SNR performance metrics. The results showed that, at a lower value of c, the SNR be better and MSE is degraded. Also From both 16 and $9.6 \mathrm{kbps}$, the ratings for $16 \mathrm{kbps}$ are better than $9.6 \mathrm{kbps}$. The work in this paper is routed towards the area of the application layer and physical layers processing to study and evaluate: The impact of implementing CELP voice codec, with 9.6kbps and 16kbps codec rate over LTE downlink PHY layer, to obtain the encoded voice bit-stream.

\section{A. Code Excited Linear Prediction Encoder Processing}

At the CELP encoder, the important aspects of voice traffic features and characteristics are represented by extracting several parameters. The basic analysis-by-synthesis CELP encoder block diagram is shown in Fig. 1. In CELP, LP is applied to estimate the vocal tract parameters by predicting the current samples of speech frame as a weighted linear combination of previous samples [12] , [14] . Linear Prediction analysis (LP-analysis) is implemented on the framed voice signal s(n) to compute linear prediction coefficients $\hat{\mathbf{a}}(\mathbf{i} ; \mathbf{m})$ of $\mathrm{m}$ order LP-analysis filter A(z) by applying autocorrelation estimate and Levinson-Durbin algorithm (L-D) [14] . For encoding the parameters of the vocal tract with good quantization properties, the linear prediction coefficients $\hat{\mathbf{a}}\left(\mathrm{I}^{\prime} \mathrm{m}\right)$ are converted to the reflection coefficients $\hat{\mathbf{k}}(\mathbf{i} ; \mathbf{m})$, by applying inverse L-D recursion [12] . For achieving the analysis-by-synthesis concept, LP coefficients are used to form $\mathbf{m}^{\text {th }}$ - order Linear 
Prediction synthesis (LP-synthesis) filter $\ominus(\mathrm{z})$ with its transfer function is represented by [15] :

$$
\Theta(z)=\frac{1}{A(z)}=\frac{1}{1+\sum_{i=1}^{m} a_{i} z^{-i}}
$$

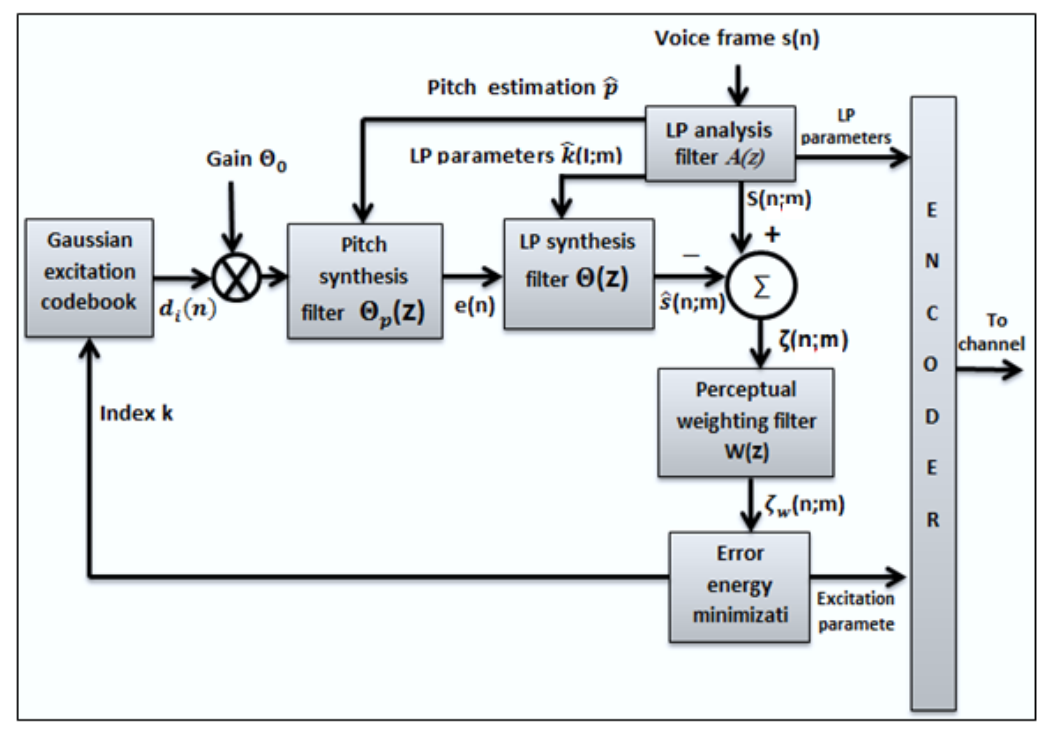

Figure 1: CELP analysis-by-synthesis coder [11]

For the voiced speech frame, the frequency of periodic pulses is referred to as the fundamental frequency or pitch. The pitch of human voices is represented with a few hundred hertz [9]. For 50 to $500 \mathrm{~Hz}$ pitch frequencies, a pitch lag, p, of 16 to 160 samples are applied. By exploiting the significant correlation from one pitch period to the next in the excitation sequence, the CELP used the long-delay correlation or pitch synthesis filter to generate the pitch periodicity of the voiced frame on a segments basis. The long-term synthesis filter transfer function is given by the formula [11] :

$$
\ominus_{p}(z)=\frac{1}{1-b z^{-p}}
$$

Where:

b: The pitch synthesis filter coefficients in the range from $0<b<1.4$ and $\mathrm{P}$ is an estimate of the number of samples in the pitch period. The search procedure for the optimal excitation sequence is typically performed on a segments basis within the frame under consideration. The excitation sequences that specified with index $\mathrm{k}$ represent the candidate vectors $d_{i}(n) s$ that are stored in the codebook and examined during the search procedure for the best sequence. A codebook of 1024 candidate sequences has been considered sufficiently large to yield good voice quality and the size of each word in the codebook is configured with 40 samples [11]. During the search procedure to generate the excitation sequence e(n), as shown in Fig. 1, the codebook sequence $d_{i}(n)$ scaled with the gain factor $\Theta_{0}$, to make the energy equal to the energy of 
the input voice, and then filter through the pitch synthesis with sequences from an interval of past excitation such that [9]:

$$
e(n)=\ominus_{0} d_{i}(n)+b e(n-p)
$$

By exciting $\Theta(z)$ filter with the obtained excitation sequence e(n), the synthesized voice frame $\hat{s}(n ; m)$ is obtained. The residual error sequence $\xi(n ; m)$ is then obtained from the difference between the synthetic voice and the original speech $s(n ; m)$ where [11] :

$$
\xi(n ; m)=s(n ; m)-\hat{s}(n ; m)
$$

To weight the mean-squared matching process within the codebook search loop, the perceptual error weighting filter W (z) is used within the CELP encoder to shapes the error such that quantization noise is partially masked by the speech spectrum envelope [14]. The perceptually weighted error sequence $\xi_{w}(n ; m)$ is obtained by further processing the obtained residual error sequence $\xi(n ; m)$ through $\mathrm{W}(\mathrm{z})$ with the coefficients of the filter that modified on a voice segments basis. The filter system function is given by [11], [14]:

$$
W(z)=\Theta(z / c) / \Theta(z)
$$

Where $\ominus(z / c)$ is the LP filter coefficients that expanded with the perceptual weighted constant, that is used to control the noise spectrum weighting [9] and the value of $\mathrm{c}$ is between 0 and 1.

\section{The Proposed CelP Codec Model Architecture}

The architecture of the proposed CELP codec model is implemented to decompose the voice signal into a set of parameters that characterize each particular frame at the encoder part; these parameters are quantized and encoded for transmission to the decoder. As part of the decoding process, the excitation parameters are extracted and the original sample is reconstructed from the extracted parameters. The model can be configured to operate at the two CELP modes: low bit rate of 9.6 kbps and medium bit rate of $16 \mathrm{kbps}$. This model is implemented as a parametric coder that is configured to operate at the two CELP modes: low bit rate of $9.6 \mathrm{kbps}$ and medium bit rate of $16 \mathrm{kbps}$ and work on any recorded or imported (.wav) sounds. For the implementation of the codebook search procedure, a Gaussian codebook CB with 1024 different candidates' vectors is generated with the size of each vector of 40 samples. These candidates' vectors were generated from a random number generator that 'seeded' at encoder and decoder with identical seeding values. Then the voice streams are handled in 20ms (160-sample) frame-based processing by the proposed CELP model. The following subsections, explain the model subsystems implementation details.

\section{A. CELP Encoder Structure}

The CELP encoder is implemented with five blocks namely: input voice frame, Linear Prediction Parameters Extraction, Excitation Parameters Extraction, parameters encoding and concatenation block as shown in Fig. 2. At the voice frame input block, the voice signal input on a frame basis and the parameter extraction and encoding processes are implemented to generate the voice frame bit-stream. The basic parameter extraction method in the CLEP model starts with the linear prediction (LP) that implement for each $20 \mathrm{~ms}$ voice frame. For extracting linear prediction coefficients of the input voice 
frame the autocorrelation is implemented using autocorrelation Simulink's predefined function block. Then the conventional Levinson-Durbin recursion is applied to the prescribed deterministic autocorrelation sequence with Simulink's predefined Levinson-Durbin block to produce the linear prediction coefficients (LPC coefficients) in vector (ar) and the reflection coefficient in vector (kpar). Then the LPC coefficients are used with the specified noise weighting factor to compute the coefficients of the filter function A (z/c) used in the perceptual weighting filter and returns them in the vector (ac).In the Excitation sequence model's block, the remainder of voice frame excitation parameters which are: the codebook index k, the gain $\ominus_{0}$, pitch delay $\mathbf{p}$ and long-delay correlation filter coefficients $\mathbf{b}$ are determined in a closed-loop search procedure to generate the best excitation sequence that results in perceptually good quality voice frame reconstructed at the decoder.

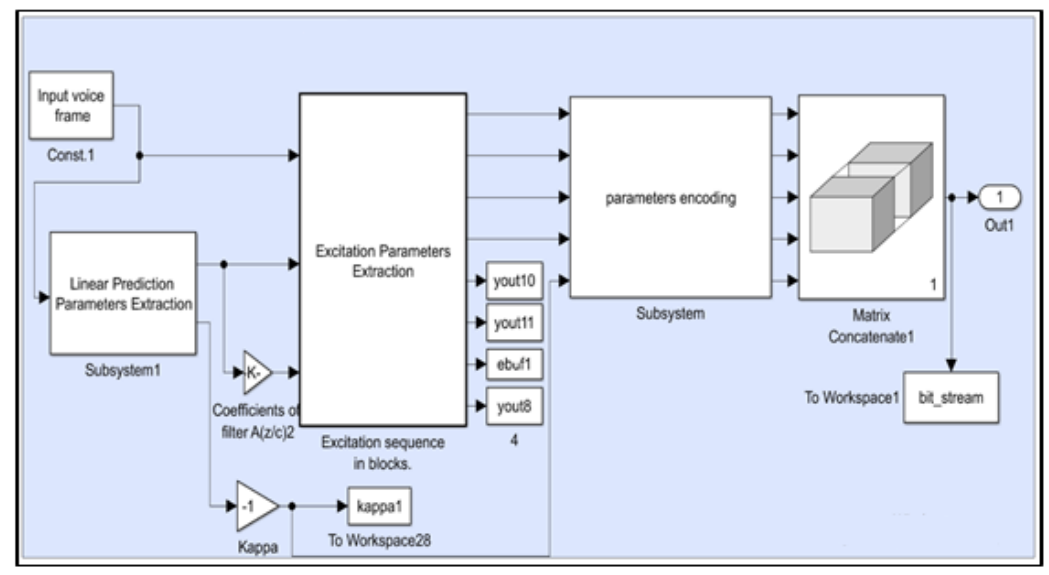

Figure 2: The implemented CELP Encoder model structure

During the search procedure, the voice frames synthesis every $5 \mathrm{~ms}$ that yield in 40 -sample voice segment. The excitation source for the given voice segment is assumed to consist of the linear combinations of the random signal (from the codebook) that is amplified by $\Theta_{0}$ and the delayed version of the excitation source due to memory hangover in the pitch filter corresponding to the previous voice segment. The vector of the previous excitation samples is stored in a vector (ebuf1) to be used for the next voice segment, and the length of this vector must be at least the max possible pitch lag. The excitation source for each voice segment is then passed through the LP-synthesis filter that is implemented using Eq. 1, to model every voice segment. The output is the synthesized voice segment which is compared with the input voice segment to constitute the error signal, which is spectrally weighted by applying it to the perceptual weighting filter W(z) of Eq. 5 . corresponding to the specified noise weighting factor to emphasize perceptual important frequencies. The code vector index and the other excitation parameters that produce the best-filtered output and results in the smallest perceptual error value are chosen to represent the input voice frame. The reflection coefficients and the excitation parameters corresponding to the voice frame are encoded for transmission as CELP bit-stream. In this model, the Integer-to-Bit conversion Simulink's block from the Communications System Toolbox has been used for each voice frame parameter to map the vector of integer values inputs to a vector of bits corresponding to the specified number of bits per integer. All of the bits of excitation 
parameters are concatenated and packed together, at the concatenation block, to construct the CELP bit-stream that is transmitted to the decoder.

\section{B. CELP Decoder structure}

At the decoder, the transmitted encoded parameters are used to recreate a frame of decoded voice. The decoder is mainly designed with selectors, parameters decoding and LP-synthesis and pitch synthesis filters blocks as shown in Fig. 3.

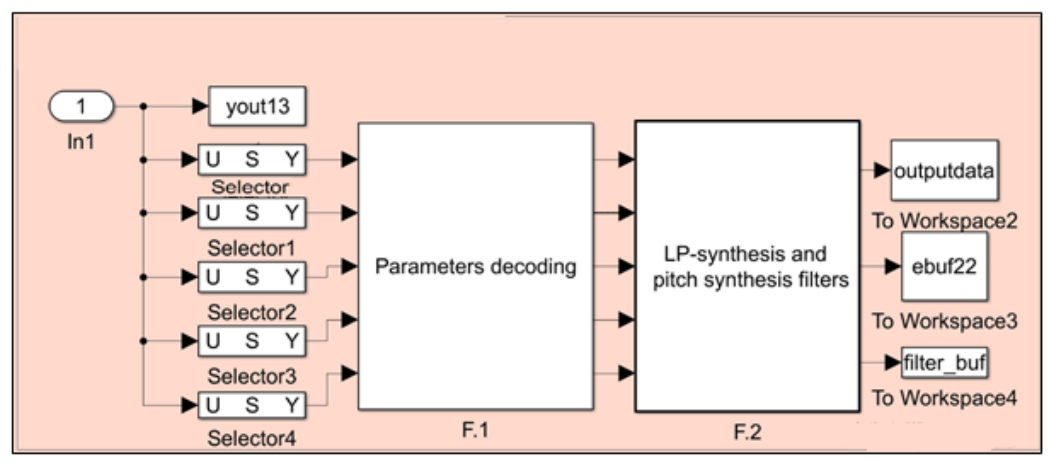

Figure 3: The implemented CELP decoder model structure

In this model the decoding process is implemented as follow:

The received bit-stream first split into five vectors, corresponding to the encoded excitation parameters, using the selectors and then decoded, at the parameters decoding block, using bits to integer converter Simulink's blocks to map the vector of bits to a corresponding vector of integer values of the reflection coefficients (par), the codebook index (k), the gain $\left(\Theta_{0}\right)$, the long correlation coefficients (b) and the lag of the pitch filter (p). For each $5 \mathrm{~ms}$ voice segment, the best candidate vector that it represents with the index $\mathrm{k}$ is chosen from the Gaussian codebook, and then scaled with $\Theta_{0}$ and filtered through $\ominus_{p}(z)$ that is implemented with coefficients $\mathrm{b}$ and lag $\mathrm{p}$ corresponding to that segments. Also, the vector of previous excitation samples is stored in a vector (ebuf2) to be used for reconstructing the next voice segment. The excitation sequence is used to excite the vocal-tract filter that is implemented with coefficients obtained from the kpar coefficients. The output of the filter is the reproduced voice waveform corresponding to the input voice frame.

\section{Channel Capacity Requirements for Celp Excitation Parameters}

The extracted voice signal parameters at the encoder are quantized and transmitted to the decoder. Each parameter has an associated channel capacity requirement corresponding to the applied CELP mode. For the two applied CELP modes, the codebook has 1024 sequences which require 10 bits to send the chosen sequence's index $\mathrm{k}$ and a total of 40 bits for each voice frame which contributes with $2 \mathrm{kbps}$ of the codec's bitrate. Also, the lag of the pitch filter, $\mathrm{P}$, is searched in the range 16 to 160 which requires 8 bits to be encoded, and a total of 32 bits. Therefore, it contributes with $1.6 \mathrm{kbps}$ of the codec's bitrate. When setting the CELP mode to a medium 16kbps bit rate, 12 bits are required for encoding each of the 12 reflection coefficients which results in a total of 144 bits per voice frame and the associated channel capacity 
requirement becomes $7.2 \mathrm{kbps}$ of voice codec bitrate. While the gain, $\Theta_{0}$, and long correlation coefficients,b, each one encoded with 13 bits per $20 \mathrm{~ms}$ and a total of 52 bits which contribute to $2.6 \mathrm{kbps}$ of the codec's rate. When CELP mode is set to a low $9.6 \mathrm{kbps}$ bit rate, 6 bits are required for encoding each of the 10 reflection coefficients and result in a total of 60 bits per $20 \mathrm{~ms}$, the associated channel capacity requirement becomes $3 \mathrm{kbps}$ of voice codec bitrate. While the gain and the long correlation coefficients are encoded with 7 and 8 bits per $20 \mathrm{~ms}$ respectively. This results in a total of 28 and 32 bits which contribute with 1.4 and 1.6 kbps of the codec's rate respectively.

\section{Voice Quality Evaluation}

This scenario quantifying the objective voice quality based on low and medium CELP codec mode by perceiving the difference between the original input and the reconstructed voice signal over various LTE link configuration parameters The elementary performance metrics considered in this scenario are SNR, segmental SNR, and mean-squared error (MSE) objective speech metrics. Table I presents the general scenario parameters.

TABLE I

Voice Quality Evaluation Model Parameters

\begin{tabular}{|c|c|c|}
\hline Type & Parameter & Value \\
\hline \multirow{3}{*}{ Voice Codec Parameters } & codec data rate & $9.6 \mathrm{Kbps}$ \\
& & $16 \mathrm{Kbps}$ \\
\cline { 2 - 3 } & Noise weighting factor & 0.65 \\
& & 0.85 \\
\cline { 2 - 3 } & System stopping criteria: & Maximum number of possible errors \\
\hline \multirow{2}{*}{ eNodeB Parameters } & channel bandwidth: & $5 \mathrm{MHz}$ \\
\cline { 2 - 3 } & DCI-symbol: & 1 -symbols \\
\hline Channel parameters & Channel models & EPA 5hz \\
\hline & Signal power & $20 \mathrm{~dB}$ \\
\hline \multirow{2}{*}{ UE parameters } & Detection mode: & MMSE \\
\cline { 2 - 3 } & Channel estimation type & Pilot-assisted Interpolation-based \\
\hline
\end{tabular}

Methodology: As input, a (Handel. mat) MATLAB file is used to create a WAVE file containing PCM data, with a single channel (mono), 16 bits per sample and $8 \mathrm{KHz}$ Sampling rate. The voice samples are input to the CELP codec model on a frame basis. Considering 50-frames of the voice signal transmit under the slow fading channel, Extended Pedestrian A (EPA), over $5 \mathrm{MHz}$ channel bandwidth, $2 \times 2$ Transmit Diversity (TD) and Channel Quality Indicator (CQI) index =6 considering signal power of $20 \mathrm{db}$. The voice samples are input to the CELP encoder and received at the CELP decoder on a frame basis.

Results: Table II presents the effect of applying the two CELP codec rates considering two values of the noise weighting factor. For the two codec bit rates, it is analyzed that at 0.65 value of noise weighting factor, better SNR values and MSE values are achieved. This is due to the more expansion in the bandwidth of the formants which became less sensitive to quantization error. In addition, at the 0.65 weighting factor value, the better SNR and MSE results were obtained for the medium codec mode of $16 \mathrm{Kbps}$ compared to the low codec mode of $9.6 \mathrm{Kbps}$.

TABLE II

SNR and MSE Measurement for $16 \mathrm{kbps}$ and $9.6 \mathrm{kbps}$ Synthesized Speech

\begin{tabular}{|c|c|c|c|c|}
\hline Noise Weighting & \multicolumn{2}{|c|}{ Low mode 9.6 Kbps } & \multicolumn{2}{c|}{ Medium mod 16 Kbps } \\
\cline { 2 - 5 } factor (c) & SNR & MSE & SNR & MSE \\
\hline $\mathrm{c}=0.65$ & -7.17839 & 0.082963 & 20.68233 & 0.14069 \\
\hline $\mathrm{c}=0.85$ & -0.1819 & 0.09398 & -2.23819 & 0.06597 \\
\hline
\end{tabular}


Fig. 4 shows the segmental SNR result which relates the ratio of the segmental signal energy to the segmental noise energy, computed over $20 \mathrm{~ms}$ voice frame for the two-codec mode considering the 50 voice frames. Also, Fig. 5 shows the quality of synthesized voice frames which is determined by observing how the reconstructed voice samples are approximated to the original voice samples. Therefore, as observed from the graphs, acceptable voice quality is well maintained in CELP for the two applied CELP modes with slightly better performance of $16 \mathrm{Kbps}$ codec compared to $9.6 \mathrm{Kbps}$ codec as it maintained better envelope replication of the original signal.

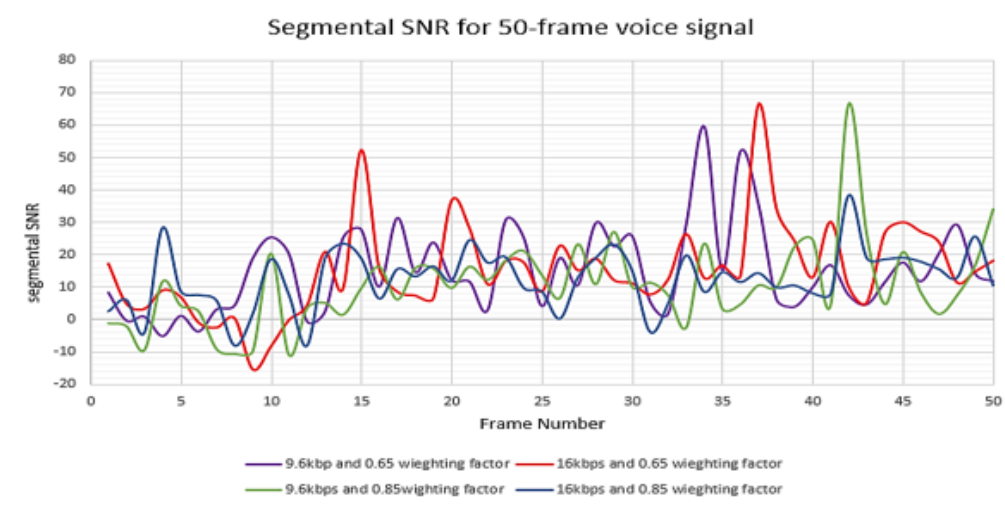

Figure 4: The segmental SNR for 9.6Kbps and 16Kbps over two noise weighting factors

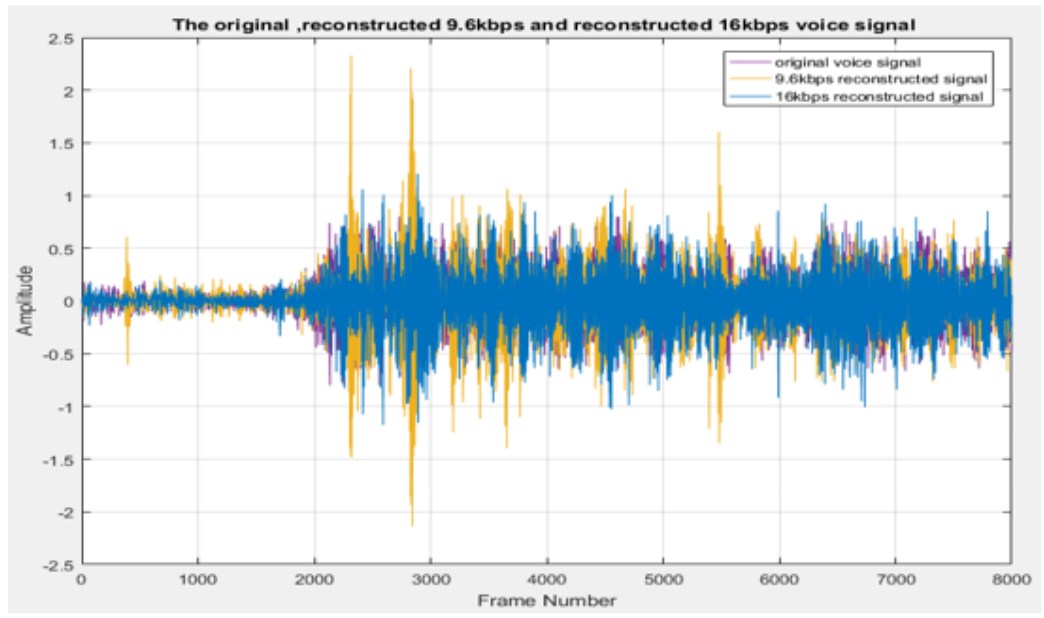

Figure 5: The original and reconstructed 50-frame voice signals

\section{Voice Capacity Evaluation}

This scenario is motivated to study the aspects of downlink LTE voice capacity for various CQIs corresponding to the 9.6 Kbps and $16 \mathrm{Kbps}$ CELP codec modes over various link configuration approach. The scenario is applied for the same input file used in the previous section considering the following basic assumptions: The general scenario model parameters are presented in Table III. 
TABLE III

Voice Capacity Evaluation Model Parameters

\begin{tabular}{|c|c|c|}
\hline Type & Parameter & Value \\
\hline \multirow{3}{*}{ Voice Codec Parameters } & codec data rate & $9.6 \mathrm{Kbps}$ \\
& & $16 \mathrm{Kbps}$ \\
\cline { 2 - 3 } & Noise weighting factor & 0.65 \\
\cline { 2 - 3 } & System stopping criteria: & Maximum number of possible errors \\
\hline \multirow{2}{*}{ eNodeB Parameters } & channel bandwidth: & $5 \mathrm{MHz}$ \\
\cline { 2 - 3 } & DCI-symbol: & 1 -symbols \\
\hline \multirow{2}{*}{ Channel parameters } & Channel models & EPA 5hz \\
& Signal power & $10 \mathrm{~dB}$ \\
\hline \multirow{2}{*}{ UE parameters } & Detection mode: & MMSE \\
\cline { 2 - 3 } & Channel estimation type & Pilot-assisted Interpolation-based \\
\hline
\end{tabular}

Methodology: in this section, the voice capacity is evaluated for all the CQI indices with the related modulation and coding scheme. The computation is applied for two-channel bandwidth deployments $5 \mathrm{MHz}$ and $10 \mathrm{MHz}$ over $2 \times 2 \mathrm{TD}$ and $2 \times 2$ Open Loop Spatial Multiplexing (OLSM).

Results: Fig. 6 and Fig. 7 show the computed average voice capacity over $5 \mathrm{MHz}$ and $10 \mathrm{MHz}$ bandwidth for the two CELP codec modes as a function of CQI indices. As depicted in the two figures, the voice capacity increases as the CQI index increases as a result of increasing the number of bits in the available RB for higher-order modulation selection and its related high coding rate. However, the voice capacities are saturated for specific CQI values this is due to the LTE restriction of assigning at least one pair of a resource block for each UE. In Fig. 6 for 5MHz bandwidth and over TD and OLSM, voice capacity saturated at CQI5 for $9.6 \mathrm{Kbps}$ while voice capacity saturated at CQI7 for 16Kbps. In Fig. 7 for $10 \mathrm{MHz}$ bandwidth and over TD and OLSM, voice capacity saturated at CQI4 for 9.6Kbps while voice capacity saturated at CQI6 for 16Kbps. From the capacity saturation, it can also be seen that the maximum number of users can be served in $5 \mathrm{MHz}$ channel bandwidth are 500 and 1000 users per cell for TD and OLSM respectively. While for 10MHz channel bandwidth are 1000 and 2000 users per cell for TD and OLSM respectively.

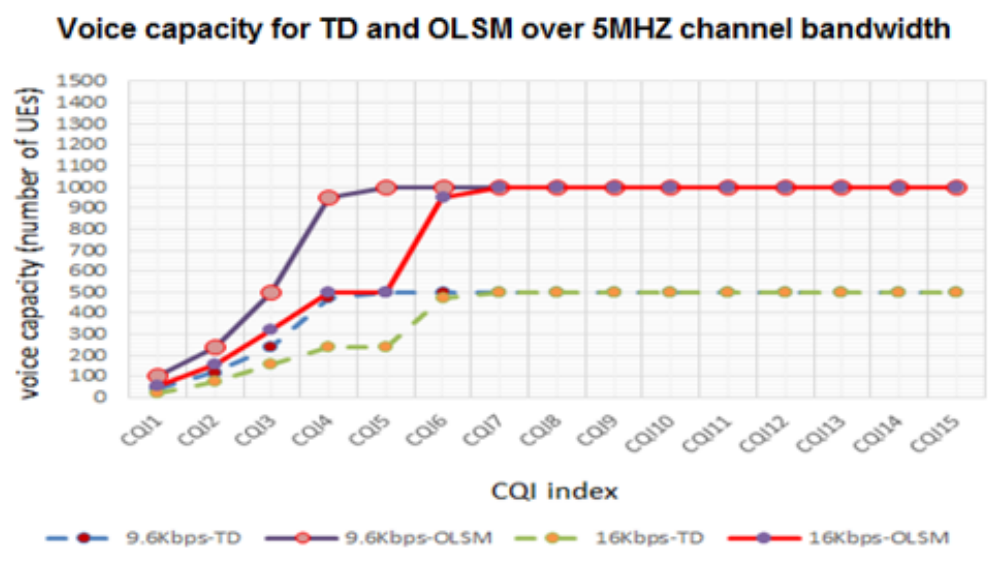

Figure 6: Voice capacity over CQI indices at $5 \mathrm{MHz}$ channel bandwidth 


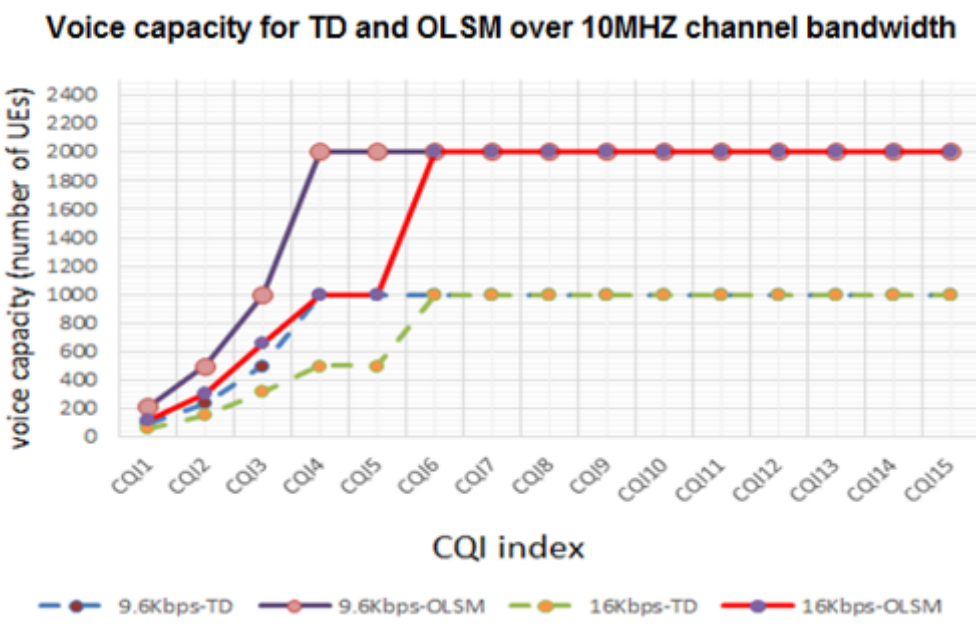

Figure 7: Voice capacity over CQI indices at $10 \mathrm{MHz}$ channel bandwidth

\section{Vi. Conclusion}

For voice quality evaluation, the examination showed better voice quality obtained with a low value of noise weighting factor and for a higher codec rate. Thus, the voice quality to voice capacity relation revealed that such a low rate codec of 9.6kbps provide better voice capacity at the cost of inferior voice quality compared to high rate codecs which show the conflicting result. For voice capacity evaluation, the investigation showed that the configuration of the link and the applied CELP codec mode mainly influence the obtained voice capacity as follow:

- For the codec part performance, the evaluation showed that the lower bit rate codec rate (9.6Kbps) provides an improvement in the capacity compared to the higher codec rate (16Kbps).

- The configuration of the system with a higher CQI index, with it, related higher-order modulation and coding scheme, resulted in increasing the capacity gain, however, this gain is increased to saturated value at specific CQI index depending on the applied transmission bandwidth and the applied MIMO configuration scheme. 
Iraqi Journal of Information and Communications Technology(IJICT)

Conference Series: The $1^{\text {st }}$ Conference of Applied Researches

in Information Engineering(ARIE2021), 2021

ISSN:2222-758X

e-ISSN:2789-7362

\section{REFERENCES}

[1] O. Amoretti,"Unleashing VoLTE Capabilities Assessing the Migration from CS Voice to IMS-Based Voice over LTE (VoLTE) " , GRIN Verlag, Vienna, 2016.

[2] D. H. Nguyen, "Enhancing and Improving Voice Transmission Quality over LTE Network: Challenges and Solutions" , PhD Thesis , Institut national des telecommunications, 2017.

[3] B. Furht and S. Ahson, "Long Term Evolution: 3GPP LTE Radio and Cellular Technology" , Taylor \& Francis Group, United State, 2009.

[4] 3GPP TR 36.913, "Requirements for Further Advancements for E-UTRA, LTE-Advanced" , Apr. , 2011.

[5] Rysavy Research, "LTE to 5g :Cellular and Broadband Innovation", white paper-5G Ammericans, 2017.

[6] M. Ray, M. Chandra, and B. P. Patil, "Speech Coding Techniques for VoIP Applications: A Technical Review" , World Applied Sciences Journal, Vol. 33, No. 5, pp. 736-743,2015.

[7] E. Dahlman, S. Parkvall, and J. Skold, "4G, LTE-Advanced Pro and the Road to 5G" , Academic Press, 2016.

[8] E. M. Mahmoudand ,T. A. Elgarf, A. A. Elhafez and A. E. Zekry, "Implementation and Evaluation of Variable Bit Rates CELP Coder" , Seventh International Conference on Computer Engineering \& Systems (ICCES) ,pp. 75-80, IEEE, 2012.

[9] M. Mulye, S. Jagtap, "Overview of Code Excited Linear Predictive Coder" , International Journal of Recent Development in Engineering and Technology(IJRDET), Vol. 9, pp. 29-36, 2014.

[10] R. Jage and S. Upadhya," Implementation of CELP and MELP Speech Coding Techniques" , International Journal of Advanced Research in Computer and Communication Engineering (IJARCCE), Vol. 5, ,pp. 702-708, 2016.

[11] M. Tandel, V. Shah, and B. Patel, "Implementation of CELP Coder and to Evaluate the Performance in Terms of Bit Rate, Coding Delay and Quality of speech" , 3rd International Conference on, Vol. 6. PP. 86-89, IEEE, 2011.

[12] S. Joshi, H. Purohit, and R. Choudhary, "A Simulation-Based Comparison on Code Excited Linear Prediction (CELP) Coder at Different Bit Rates" , Proceedings of International Conference on Recent Advancement on Computer and Communication, Springer, Singapore, pp. 297-304 ,2018.

[13] H. Mohammed, K. H. Bilal and. M. A. Hassan, "Voice over IP over LTE Network: A Review" , ijem, Vol. 28, Issue 01 ,PP. 1-7, 2015.

[14] K. N. Ramamurthy and A. S. Spanias , "MATLAB Software for the Code Excited Linear Prediction Algorithm :The Federal Standard-1016" , Synthesis Lectures on Algorithms and Software in Engineering, pp. 1-109,2010.

[15] I. Mcloughlin, "Applied Speech and Audio Processing: with Matlab Examples" , Cambridge University Press, 2009. 